\title{
ASSOCIAÇÃO ENTRE IMC E TESTE DE COORDENAÇÃO CORPORAL PARA CRIANÇAS (KTK). UMA META-ANÁLISE
}

ASSOCIATION BETWEEN BMI AND BODY COORDINATION TEST FOR CHILDREN (KTK). A META-ANALYSIS

ASOCIACIÓN ENTRE IMC Y TEST DE COORDINACIÓN CORPORAL PARA NIÑOS (KTK). UN META-ANÁLISIS

\author{
Leonardo Gomes de Oliveira Luz ${ }^{1,2}$ \\ (Educador Físico) \\ André Filipe Teixeira e Seabra ${ }^{3}$ \\ (Educador Físico) \\ Rute Santos $^{3}$ (Educadora Física) \\ Cristina Padez ${ }^{4}$ (Antropóloga) \\ José Pedro Ferreira ${ }^{2}$ \\ (Educador Físico) \\ Manuel João Coelho-e-Silva² \\ (Educador Físico)
}

1. Universidade Federal de Alagoas, Laboratório de Cineantropometria, Atividade Física e Promoção da Saúde - LACAPS, Arapiraca, Alagoas, Brasil.

2. Universidade de Coimbra, Centro de Investigação do Desporto e Atividade Física, Coimbra, Portugal. 3. Universidade do Porto, Centro de Investigação em Atividade Física, Saúde e Lazer (CIAFEL),

Porto, Portugal.

4. Universidade de Coimbra, Centro de Investigação em Antropologia e Saúde (CIAS), Coimbra, Portugal.

\section{Correspondência:}

Rua Vale das Flores, 43, 1B. Coimbra, Portugal. 3030-486.

leonardoluz.ufal@gmail.com

\section{RESUMO}

O Teste de Coordenação Corporal para Crianças (Körperkoordinationstest für Kinder, KTK) tem sido utilizado na avaliação da coordenação motora de crianças e adolescentes. O objetivo desta revisão sistemática com meta-análise foi analisar a relação entre o índice de massa corporal (IMC) e o desempenho motor no KTK em crianças e jovens escolares saudáveis. O estudo baseou-se em pesquisas indexadas nas bases eletrônicas PubMed e SciELO. Os descritores foram: "Körperkoordinationstest für Kinder" " "KTK". Foram considerados artigos em inglês e português publicados até Outubro de 2014. A qualidade dos estudos foi determinada pelas Escalas PEDro e STROBE. Foi realizada meta-análise utilizando o software Comprehensive Meta-Analysis V2. Foram incluídos 10 estudos. O resultado global com base no modelo de efeitos fixos mostrou que valores maiores de IMC estão diretamente associados ao baixo desempenho no KTK. Apesar da pequena diferença entre ambos, a magnitude do efeito da associação foi classificada como pequena, porém, significativa para o sexo masculino ( $r=0,29 ;$ IC95\% 0,27 a 0,32; $Z=22,47 ; p=0,000)$ e moderada para o sexo feminino ( $r=0,32 ; \mathrm{IC} 95 \% 0,30$ a 0,$34 ; Z=24,76 ; p=0,000)$. Os estudos apresentaram características amostrais bastante divergentes quanto ao tamanho da amostra e a faixa etária dos sujeitos, além de nenhum deles ter analisado o estado maturacional dos participantes. Conclui-se que há uma tendência de relação positiva entre valores elevados de IMC e o baixo desempenho no KTK. Para confirmação desta tendência, recomenda-se que os próximos trabalhos considerem o estado maturacional dos participantes, além de possibilitarem uma análise por sexo e idade.

Palavras-chave: meta-análise, adolescente, antropometria, atividade motora.

\section{ABSTRACT}

The Body Coordination Test for Children (Körperkoordinationstest für Kinder, KTK) has been used in the evaluation of motor coordination in young people. The objective of this systematic review and meta-analysis study was to analyze the relationship between body mass index (BMI) and the motor performance by the KTK test in healthy children. The study was based on research indexed in the electronic databases PubMed and SciELO. The descriptors were "Körperkoordinationstest für Kinder" and "KTK". Studies published in English and Portuguese until October 2014 were considered. The quality of the studies was determined by PEDro and STROBE scales. Meta-analysis was performed using the Comprehensive MetaAnalysis V2 software. A total of 10 studies were included. The overall result based on the fixed effects model showed that higher values of the BMI are directly associated with low performance in the KTK. Despite the small difference between genders, the magnitude of the association effect was classified as small, but significant for males ( $r=0.29 ; 95 \% \mathrm{Cl} 0.27$ to $0.32 ; Z=22.47 ; p=0.000$ ) and moderate for females $(r=0.32 ; 95 \% \mathrm{Cl} 0.30$ to $0.34 ; Z=24.76, p=0.000)$. Studies have shown different characteristics as the sample size and the age of the subjects, and none of them have analyzed the maturational status of the participants. Based on these results, there is a trend of a positive relationship between high values of BMI and low performance in the KTK.

Keywords: meta-analysis, adolescent, anthropometry, motor activity.

\section{RESUMEN}

El "Körperkoordinationstest für Kinder" (KTK) ha sido utilizado en la evaluación de la coordinación motriz de niños y adolescentes. El objetivo de esta revisión sistemática con meta-análisis fue analizar la relación entre el índice de masa corporal (IMC) y el desempeño en el KTK en niños y jóvenes escolares saludables. El estudio se basó en investigaciones indexadas en las bases de datos electrónicas PubMed y Scielo. Los descriptores fueron "Körperkoordinationstest für Kinder" " "KTK". Se consideraron los artículos en Inglés y portugués publicados hasta octubre de 2014. La calidad de los estudios se determinó por Escalas PEDro y STROBE. Se realizó un meta-análisis con el uso del software Comprehensive Meta-Analysis V2. Se incluyeron 10 estudios. El resultado global basado en el modelo de efectos fijos mostró que valores más altos de IMC están directamente asociados al bajo desempeño en el KTK. A pesar de la pequeña diferencia entre ambos, la magnitud del efecto de la asociación fue clasificada como pequeña, pero significativa para el sexo masculino $(r=0,29 ; 95 \% \mathrm{Cl} 0,27-0,32 ; Z=22,47, p=0,000)$ y moderada para el sexo femenino $(r=0,32 ; 1 C$ 95\% 0,30-0,34; $Z$ $=24,76, p=0,000$ ). Los estudios presentaron características muestrales bastante divergentes cuanto al tamaño de la 
muestra y el grupo de edad de los sujetos, además de haberse analizado el estado de madurez de los participantes. Se concluye que hay una tendencia de relación positiva entre los valores elevados de IMC y el bajo desempeño en el KTK. Para la confirmación de esta tendencia, se recomienda que los futuros estudios consideren el estado de madurez de los participantes, además de posibilitar un análisis por sexo y edad.

Palabras clave: meta-análisis, adolescente, antropometría, actividad motriz.

\section{INTRODUÇÃO}

Janssen e Leblanc ${ }^{1}$, numa revisão sistemática, constataram evidências associando a prática de atividades físicas na infância e adolescência a inúmeros benefícios de saúde, nomeadamente a saúde cardiovascular, metabólica, esquelética e mental, além de auxiliar no controle ponderal, reduzindo o risco de obesidade. A literatura parece associar a importância da prática de atividade física para a saúde dos jovens ao desenvolvimento dos componentes da aptidão física relacionada com a saúde².

Em indivíduos jovens, assim como para os adultos, os benefícios da prática regular de atividade física sobre a aptidão física relacionada à saúde são tópicos bastante estudados na literatura científi$\mathrm{ca}^{3,4}$. Estudos têm demonstrado que a prática de atividade física é uma componente fundamental de um estilo de vida saudável ${ }^{5}$, na medida em que promove benefícios sobre a atividade cognitiva ${ }^{6}$, a estrutura óssea ${ }^{2,7}$, a saúde metabólica ${ }^{8}$ e o sistema cardiovascu$\operatorname{lar}^{9}$. A atividade física na infância e na adolescência está também associada à manutenção da atividade física em idades subsequentes da vida ${ }^{10}$ e na fase adulta ${ }^{11}$.

Existe na literatura o renascer de uma preocupação com o desenvolvimento da coordenação motora e as implicações desta capacidade na saúde das crianças e dos adolescentes. A coordenação motora pode ser definida como a interação harmoniosa e econômica do sistema musculoesquelético, do sistema nervoso e do sistema sensorial com a finalidade de produzir ações motoras precisas e equilibradas ${ }^{12}$. Inúmeras evidências têm mostrado associação da coordenação motora com aspectos relacionados à saúde de crianças ${ }^{13-15}$. Dentre os aspectos estudados, destacam-se a aptidão física relacionada com a saúde ${ }^{16,17}$, o nível de atividade física $^{15,18}$, a morfologia corporal ${ }^{19}$, a maturação biológica ${ }^{20}$ e as características sociodemográficas ${ }^{21}$. Ainda, estudiosos mencionam que o desenvolvimento da coordenação motora na infância poderá influenciar decisivamente na motivação e no envolvimento em práticas motoras, relacionando-se positivamente com a realização de atividades físicas e com a participação desportiva ${ }^{10,22}$, além de, também, aumentar a probabilidade de obtenção de melhores desempenhos acadêmicos ${ }^{23}$.

Segundo Cools et al. ${ }^{24}$, vários são os instrumentos de avaliação da coordenação motora, dentre os quais, citam-se: teste de integração sensorial da Califórnia do Sul, teste de Bruininks-Oseretsky de proficiência motora, teste de habilidades de crianças jovens, teste de sensibilidade cinestésica, exame da criança com disfunção neurológica menor, teste de desenvolvimento motor grosso, bateria de avaliação de movimento para crianças - teste do movimento $A B C$, teste de coordenação corporal para crianças - Körperkoordinationstest für Kinder - KTK, entre outros.

Teste de Coordenação Corporal para Crianças - Körperkoordinationstest für Kinder (KTK): surgiu frente à necessidade de diagnosticar mais sutilmente as insuficiências motoras em crianças com lesões cerebrais e/ ou desvios comportamentais. O desenvolvimento do teste ocorreu na
Alemanha, durante cinco anos de estudos, em diversos estágios, e culminou com sua concepção final publicada em $1974^{25}$.

O KTK abrange componentes da coordenação corporal como: o equilíbrio, o ritmo, a força, a lateralidade, a velocidade e a agilidade. O teste leva cerca de 10 a 15 minutos para ser administrado a cada criança ou adolescente entre os 5 e os 14 anos de idade. A sala de teste deve apresentar uma dimensão mínima de 4×5 metros. O KTK tem, em sua forma final, quatro tarefas: equilíbrio em marcha à retaguarda, saltos monopedais, saltos laterais e transposição lateral. O resultado de cada item é comparado com os valores normativos fornecidos pelos autores, sendo-Ihe atribuído a cada item um quociente. O somatório dos quatro quocientes representa o quociente motor $(\mathrm{QM})$, que pode ser apresentado em valores percentuais ou absolutos, permitindo classificar as crianças, para cada idade e sexo, segundo o seu nível de desenvolvimento coordenativo: (1) perturbações da coordenação (QM < 70); (2) insuficiência

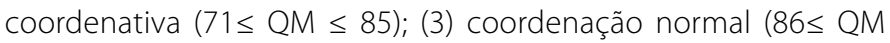

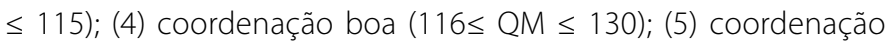
muito boa (131 $\leq \mathrm{QM} \leq 145)$. O KTK é um instrumento altamente confiável e válido e, portanto, frequentemente usado para avaliar a coordenação motora em crianças ${ }^{26,27}$.

Mais recentemente, o KTK tem sido utilizado como teste para avaliação da coordenação motora em diversos estudos realizados com crianças e adolescentes aparentemente saudáveis ${ }^{27,28}$. Destes, destacam-se, pelo seu maior número, os trabalhos que tiveram como objetivo associar o desempenho motor na bateria de testes do KTK com o índice de massa corporal (IMC) de crianças e jovens. Apesar do maior número de estudos relacionados com esse tema, percebe-se na literatura uma carência quanto à real magnitude do efeito dos resultados reportados.

Uma recente revisão sistemática sobre a relação entre a coordenação motora e aspectos da saúde de crianças e adolescentes mostrou que o tamanho corporal pode influenciar no desempenho da coordenação motora ${ }^{29}$. Contudo, esta relação não esteve presente em todos os estudos analisados.

A presente revisão sistemática com meta-análise tem interesse, especificamente, nos estudos que utilizaram o KTK como instrumento para avaliar a coordenação motora de crianças e jovens, principalmente pelos seguintes aspectos: 1) trata-se de um teste apropriado para crianças com desenvolvimento motor típico padrão, bem como para crianças com danos cerebrais, problemas comportamentais ou dificuldades de aprendizagem; 2) o teste engloba uma faixa etária de 5 a 14 anos de idade, ou seja, pode ser aplicado em crianças e adolescentes; 3) o KTK é fácil de configurar e leva pouco tempo para administrar; 4) o KTK é um dos testes de menor custo de execução; 5) o teste é completamente padronizado e considerado altamente confiável24. Aliado às questões mencionadas anteriormente, que denotam vantagens do KTK em relação a outros testes de avaliação do desempenho motor, outro ponto importante, que merece destaque, é o fato do KTK ser o 
teste mais comumente utilizado em estudos que associam o IMC com o desempenho de crianças em teste de coordenação motora. Diante do exposto, o objetivo desta revisão sistemática seguida de meta-análise é analisar a relação entre o IMC e o desempenho no KTK em crianças e jovens escolares saudáveis.

\section{MÉTODOS}

A presente revisão baseou-se na realização de uma pesquisa e identificação de artigos publicados sobre o tema e indexados na bases eletrônicas PubMed e Scielo. Para busca online, os termos descritores utilizados foram: "Körperkoordinationstest für Kinder" e "KTK", tendo em vista que o objetivo inicial foi de encontrar toda a literatura disponível sobre o KTK. A compilação dos estudos decorreu de Agosto a Outubro de 2014 (data da última busca: 26/10/2014).

\section{Critérios de inclusão}

Foram incluídos estudos de intervenção e observacionais, transversais e longitudinais, realizados com escolares, crianças e adolescentes, de ambos os sexos. Os artigos analisados representam o total resultante da busca isolada de cada um dos descritores mencionados anteriormente. Foram analisadas todas as publicações nos idiomas português e inglês. Os estudos deveriam, obrigatoriamente, reportar os resultados da relação entre o IMC dos sujeitos e o desempenho no KTK.

Quanto aos critérios de exclusão adotados, o presente estudo optou por não utilizar: a) textos de revisão sobre o tema; b) estudos que não utilizaram a bateria KTK completa, com as quatro tarefas; c) estudos cuja amostra fosse de crianças com qualquer distúrbios de coordenação motora; d) estudos realizados com crianças em ambiente de treinamento desportivo e, por uma questão de controle da qualidade dos estudos incluídos, e) estudos com um escore menor do que quatro na Escala de PEDro e $80 \%$ na Escala STROBE (Strengthening the Reporting of Observational studies in Epidemiology).

Os passos utilizados na seleção dos estudos estão ilustrados na figura 1.

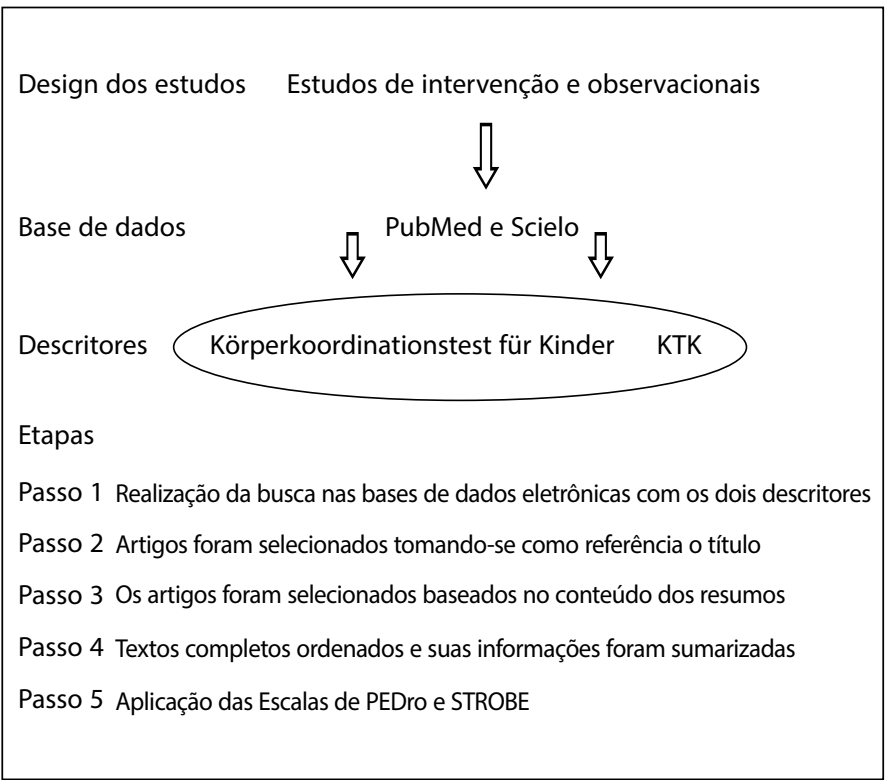

Figura 1. Diagrama de seleção dos estudos.

\section{Avaliação da qualidade metodológica dos estudos}

A qualidade dos estudos foi determinada pela utilização de dois instrumentos distintos, a Escala PEDro para os estudos de intervenção e a Escala STROBE para os estudos observacionais.
A Escala PEDro ${ }^{30}$, baseada na lista de Delphi ${ }^{31}$ e traduzida para a língua portuguesa em 2009, tem 11 itens que avaliam a qualidade metodológica dos ensaios clínicos aleatórios, observando dois aspectos do estudo: a validade interna e se contém informações estatísticas suficientes para torná-lo interpretável.

Apenas dez dos 11 critérios avaliados recebem pontuação ${ }^{30}$, ficando a primeira questão sem pontuação. Cada critério é pontuado de acordo com a sua manifestação no estudo avaliado. Caso seja presente, atribui-se um ponto e na sua ausência não se pontua. A pontuação final é obtida pela soma de todas as questões cujas respostas são positivas. De acordo com Moseley et al. ${ }^{32}$, estudos com escore igual ou maior que cinco (50\%) são considerados de alta qualidade. Apesar disso, devido à impossibilidade de se alcançar certas condições, como o cegamento dos profissionais que conduzem a intervenção ou dos sujeitos em estudos de intervenção, a pontuação máxima que poderia ser alcançada por um estudo de intervenção seria 8/10. Para a presente revisão, todos os estudos pontuados pela Escala PEDro com valores iguais ou superiores a 4 foram considerados estudos de boa qualidade metodológica e, portanto, incluídos na análise.

Já a qualidade dos estudos observacionais foi determinada pela Escala STROBE (Strengthening the Reporting of Observational studies in Epidemiology) ${ }^{33}$. A lista de verificação e os documentos que descrevem a Iniciativa STROBE foram elaborados por meio de um processo de colaboração que incluiu pesquisadores que trabalham na área de epidemiologia, estatística e metodologia de pesquisa, além de editores de diversas revistas científicas. A escala STROBE compreende uma lista de verificação de 22 itens que deveriam ser abordados na descrição dos estudos observacionais. Os itens relacionam-se com as informações que deveriam estar presentes no título, resumo, introdução, metodologia, resultados e discussão de artigos científicos que descrevem estudos observacionais ${ }^{34}$. Os estudos pontuados pela Escala STROBE com valores iguais ou maiores a 80\% do máximo possível foram incluídos na análise.

\section{Análise dos dados}

A meta-análise foi realizada com o programa Comprehensive meta analysis V2 e o resultado global com base no modelo de efeitos fixOS $^{35}$ mostra a correlação entre os valores de IMC e o baixo desempenho no KTK, além do seu intervalo de confiança a 95\%, a magnitude do efeito e a significância estatística $(p<0,05)$. Os coeficientes de correlação foram interpretados da seguinte forma ${ }^{36}$ : trivial $(r<0,1)$, fraca $(0,1<r<0,3)$, moderada $(0,3<r<0,5)$, forte $(0,5<r<0,7)$, muito forte $(0,7<r<0,9)$, e quase perfeita $(r>0,9)$. Além disso, foi realizada também uma análise de viés de publicação por meio de inspeção visual do "funil invertido". O gráfico de dispersão no formato de "funil invertido" foi utilizado para avaliar o risco de viés de publicação, por meio da avaliação da distribuição dos dados observados no gráfico. Quando não há risco de viés de publicação, obtém-se a figura que dá nome ao gráfico. Os valores obtidos no gráfico não são sistematicamente examinados e a simetria (ou assimetria) tem geralmente sido definida por meio de inspeção visual. Relativamente às estatísticas de heterogeneidade, utilizou-se o Q de Cochran para o cálculo do $1^{2}$ de Higgin, que representa a percentagem de variância atribuída à heterogeneidade. O $\mathrm{I}^{2}$ toma valores entre 0\% e 100\%. Valor zero indica ausência de heterogeneidade. Os demais valores classificam-se em: baixa heterogeneidade $\left(25 \%<1^{2}<50 \%\right)$, heterogeneidade moderada $\left(50 \%<1^{2}<75 \%\right)$ e heterogeneidade elevada $\left(1^{2}>75 \%\right)^{37}$. Por último, optou-se por realizar, também, uma análise dos subgrupos masculino e feminino para melhor compreender os resultados e a heterogeneidade dos dados. 


\section{RESULTADOS}

Foram identificados 102 estudos (87 no PubMed e 15 no Scielo); 84 foram excluídos após análise dos títulos e resumos, por serem duplicados ou por não se enquadrarem nos critérios de seleção; 18 foram analisados pelo texto completo, dos quais 8 foram excluídos por não se enquadrarem nos critérios de inclusão ou por não terem atingido os 4 pontos na Escala PEDro ou os 80\% na Escala STROBE (figura 2). Dos 10 estudos selecionados, um era de intervenção (de curta duração) e nove de âmbito observacional.

Na tabela 1, encontram-se as características gerais dos estudos selecionados.

Os resultados encontrados evidenciam uma predominância de estudos realizados na Europa (nove estudos), o que já era esperado levando-se em consideração a origem do teste, com destaque para os estudos realizados na Bélgica (quatro estudos) e em Portugal (quatro estudos). Todos os estudos compilados apresentaram em sua amostra crianças de ambos os sexos, embora alguns não

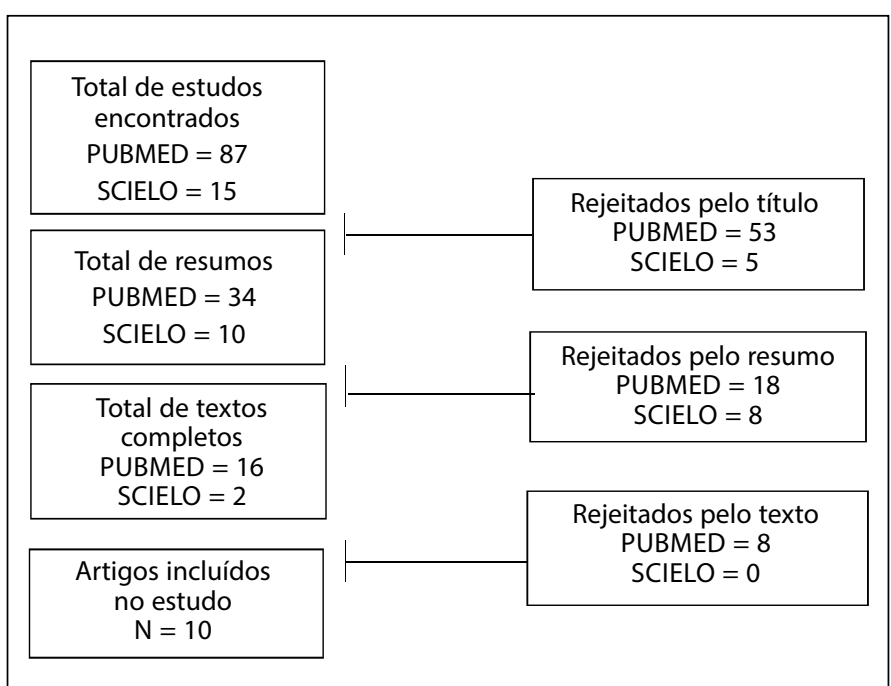

Figura 2. Sumário do fluxograma de identificação, triagem, elegibilidade e inclusão dos estudos selecionados.

Tabela 1. Dados gerais quanto à origem, dimensão e idade da amostra e principais dados estatísticos reportados dos estudos selecionados.

\begin{tabular}{|c|c|c|c|c|c|}
\hline Estudo (ano) & $\begin{array}{c}\text { Base de } \\
\text { dados }\end{array}$ & Amostra & País & $\begin{array}{l}\text { Idade } \\
\text { (anos) }\end{array}$ & $\begin{array}{c}\text { Dados } \\
\text { estatísticos }\end{array}$ \\
\hline Catenassi et al..38, 2007 & Scielo & $\begin{array}{c}27 \text { crianças } \\
(\text { meninas }=11)\end{array}$ & Brasil & 4 a 6 & Correlação e N \\
\hline D'Hondt et al. ${ }^{27}, 2011$ & PubMed & $\begin{array}{c}954 \text { crianças } \\
\text { (meninas }=500)\end{array}$ & Bélgica & 5 a 12 & $\begin{array}{c}\text { Média, desvio } \\
\text { padrão e N }\end{array}$ \\
\hline D'Hondt et al. ${ }^{39}, 2011$ & PubMed & $\begin{array}{c}72 \text { crianças } \\
\text { (meninas = 24) }\end{array}$ & Bélgica & 7 a 13 & $\begin{array}{l}\text { Média, desvio } \\
\text { padrão e N }\end{array}$ \\
\hline D'Hondt et al. ${ }^{44}, 2013$ & PubMed & $\begin{array}{c}100 \text { crianças } \\
\text { (meninas = 48) }\end{array}$ & Bélgica & 6 a 10 & Correlação e N \\
\hline D'Hondt et al. ${ }^{28}, 2014$ & PubMed & $\begin{array}{c}754 \text { crianças } \\
\text { (meninas }=49,2 \%)\end{array}$ & Bélgica & 7 a 13 & Correlação e N \\
\hline Graf et al.40, 2004 & PubMed & $\begin{array}{c}668 \text { crianças } \\
\text { (meninas = 327) }\end{array}$ & $\begin{array}{c}\text { Alema- } \\
\text { nha }\end{array}$ & $6,7 \pm 0,4$ & Correlação e N \\
\hline Lopes et al. ${ }^{15}, 2012$ & PubMed & \begin{tabular}{|c|}
7175 crianças \\
(meninas = 3559) \\
\end{tabular} & Portugal & 6 a 14 & Correlação e N \\
\hline Lopes et al.23, 2013 & PubMed & $\begin{array}{c}596 \text { crianças } \\
\text { (meninas = 281) }\end{array}$ & Portugal & 9 a 12 & $\begin{array}{c}\text { Odds ratio e } \\
\text { intervalo de } \\
\text { confiança }\end{array}$ \\
\hline Martins et al. ${ }^{14}, 2010$ & PubMed & $\begin{array}{c}285 \text { crianças } \\
\text { (meninas }=142)\end{array}$ & Portugal & 6 & $\begin{array}{l}\text { Correlação e } \\
\text { erro padrão }\end{array}$ \\
\hline Melo e Lopes², 2013 & Scielo & $\begin{array}{c}794 \text { crianças } \\
\text { (meninas = 398) }\end{array}$ & Portugal & 6 a 9 & Correlação e N \\
\hline
\end{tabular}

$\mathrm{N}$ : tamanho da amostra tenham apresentado os resultados da associação entre o IMC e o desempenho no KTK dos sujeitos analisados, separadamente, para meninos e meninas. Percebe-se uma heterogeneidade no quantitativo amostral dos estudos e a faixa etária predominante é dos 6 aos 10 anos de idade.

Os dados apresentados em texto e em tabelas são, em sua maioria, valores de correlação entre as variáveis de estudo e o tamanho da amostra.

A tabela 2 reporta os resultados da correlação e a magnitude do efeito de cada um dos estudos, separadamente, assim como o valor geral.

Dentre os estudos analisados, o resultado de Catenassi et al. ${ }^{38}$ não apresentou associação significativa ( $p=0,845)$. A magnitude da associação mostrada pelos estudos foi menor no estudo de Martins et al. ${ }^{14}$ e maior em D'Hondt et al..$^{39} \mathrm{O}$ tamanho do efeito na análise para ambos os sexos combinados foi significativo, mas sua correlação classificada como trivial ( $r=0,06 ;$ IC 95\% 0,05 a 0,06; $Z=16,21 ; p=0,000$ ). As tabelas 3 e 4 reportam os resultados da correlação e magnitude do efeito de cada um dos estudos, separadamente, assim como os valores estatísticos no geral, para meninos e meninas, respectivamente. Nos meninos, a associação mostrada pelos estudos variou de $r=0,17$ (IC 95\% 0,05 a 0,28; $Z=2,78 ; p=0,005)$, no estudo de Graf et $a l^{40}$, a $r=0,72$ (IC 95\% 0,63 a 0,79; Z=10,36; $p=0,000)$, em D'Hondt et al. ${ }^{39}$. A associação encontrada na análise dos seis estudos, entre o IMC e o desempenho no teste KTK em sujeitos do sexo masculino, foi de $r=0,29$ (IC 95\% 0,27 a 0,32; Z=22,47; $p=0,000$ ), ou seja, uma associação fraca, porém significativa, entre o valor mais alto de IMC e a di-

Tabela 2. Estudos, valores de correlação e magnitude do efeito dos seus resultados e valor representativo geral da relação entre o IMC e o baixo desempenho no KTK de escolares de ambos os sexos.

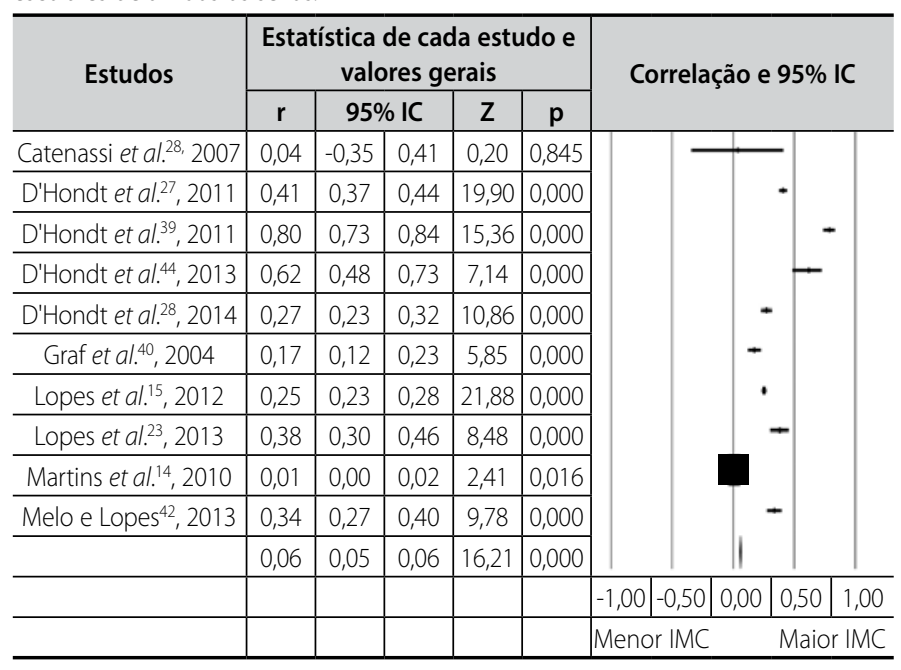

Tabela 3. Estudos, valores de correlação e magnitude do efeito dos seus resultados e valor representativo geral da relação entre o IMC e o baixo desempenho no KTK de escolares do sexo masculino.

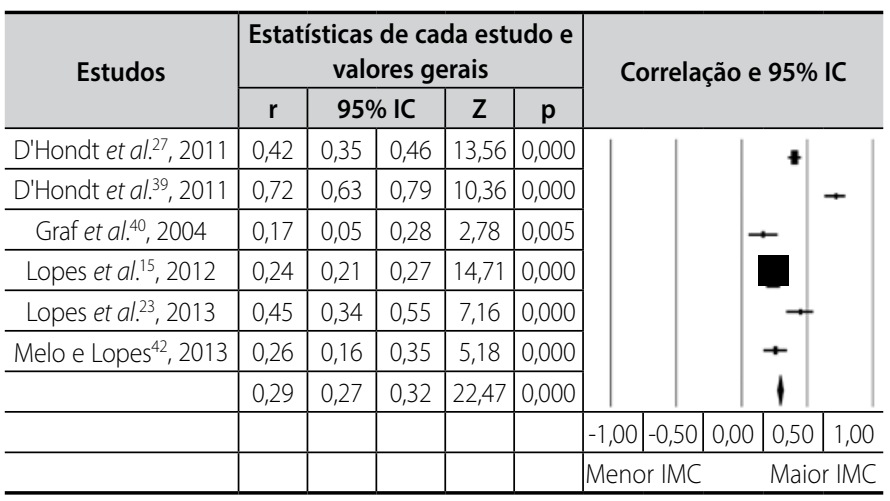


minuição no desempenho do KTK. Nas meninas, o valor encontrado foi de $r=0,32$ (IC 95\% 0,30 a 0,34), caracterizando-se por uma associação moderada, tendo-se obtido uma significativa magnitude dos efeitos com o valor de $Z=24,76(p=0,000)$.

Na figura 3, encontra-se representada a tendência do viés das publicações através do gráfico de dispersão em funil, "funil invertido" ou "árvore de natal"41. Cada ponto do gráfico representa um estudo pelo que a forma assimétrica obtida no presente estudo sugere que houve viés de publicação.

Deste modo, calculou-se o $1^{2}$ de Higgin, com base no valor do Q de Cochran, tendo a percentagem da variância atribuída à heterogeneidade atingido os $99 \%$ para a totalidade das amostras analisadas e $94 \%$ no caso dos meninos e $95 \%$ nas meninas, quando calculada separadamente por sexo.

Tabela 4. Estudos, valores de correlação e magnitude do efeito dos seus resultados e valor representativo geral da relação entre o IMC e o baixo desempenho no KTK de escolares do sexo feminino.

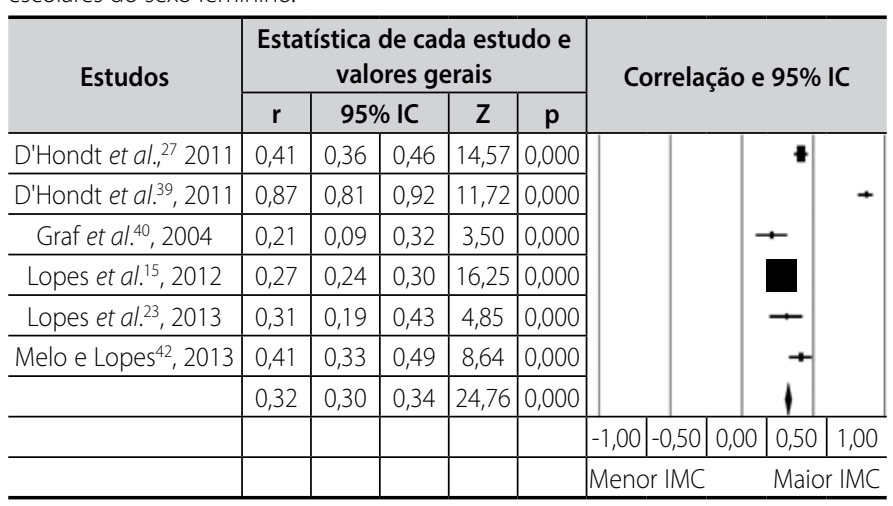

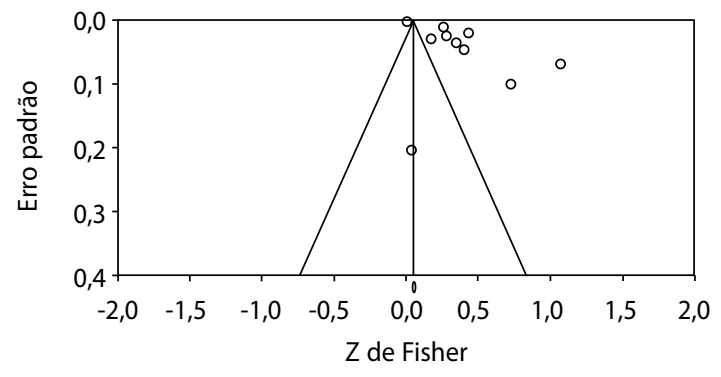

Figura 3. Resultado gráfico de dispersão em "funil invertido" do erro padrão pelo Z de Fisher.

\section{DISCUSSÃO}

Os resultados encontrados apontam para uma associação positiva entre os maiores valores de IMC e o menor desempenho nas tarefas do KTK. Para ambos os sexos, de forma combinada, a associação apresentou um valor de $r=0,06$ (IC 95\% 0,05 a 0,06), ou seja, uma relação classificada como trivial, tendo uma magnitude de efeito significativa com valor de $Z=16,21$ ( $p=0,000)$. Separadamente, a associação para o sexo feminino foi de $r=0,32$ (IC 95\% 0,30 a 0,34), com magnitude de efeito de $Z=24,76(p=0,000)$ e no sexo masculino com $r=0,29$ (IC 95\% 0,27 a 0,32) e valor de $Z=22,47(p=0,000)$. Os estudos selecionados apresentaram-se divergentes principalmente quanto às características da amostra (número de observados e faixa etária da amostra). Todos os estudos analisados incluíram participantes de ambos os sexos. No entanto, apenas seis dos 10 estudos interpretaram isoladamente os resultados para meninos e meninas $15,27,39,40,42,43$. Quanto ao quantitativo da amostra, três estudos avaliaram um total de 100 crianças ou menos ${ }^{38,39,44}$, enquanto os de- mais apresentaram uma amostra superior, tendo o estudo de maior abrangência atingido o valor de 7.175 sujeitos no total ${ }^{15}$. A faixa etária estudada variou dos 4 aos 14 anos de idade, tendo estudos concentrado a amostra em crianças pré-púberes ${ }^{14,38,40,42,44}$, enquanto outros abrangeram crianças e jovens ${ }^{15,27,28,39,43}$. Os aspectos listados são de suma importância na discussão dos resultados encontrados, uma vez que na meta-análise cada estudo exerce uma contribuição particular no resultado da correlação e na magnitude do efeito da associação global entre os escores do IMC e do KTK.

Quanto aos resultados da meta-análise para cada sexo, separadamente, percebeu-se um aumento no valor da correlação tanto para o sexo masculino, quanto para o feminino. Este resultado pode ter sido influenciado, entre outros motivos, pela exclusão do estudo de Catenassi et al. ${ }^{38}$, cuja associação entre as variáveis não teve magnitude de efeito significativa ( $Z=0,20 ; p=0,845)$. Neste estudo, os autores avaliaram apenas 27 crianças brasileiras, dos 4 os 6 anos de idade, e encontraram uma correlação trivial entre as variáveis em questão com r=0,04 (IC 95\% -0,35 a 0,41). Por outro lado, o estudo publicado por D'Hondt et al. ${ }^{39}$ cuja amostra foi composta por 72 crianças belgas, com idades variando entre os 7 e 13 anos, obtiveram uma correlação muito forte, com magnitude de efeito significativa, e permitiu as análises para ambos os sexos, separadamente, o que contribuiu para o aumento dos valores globais reportados pela meta-análise.

Essas diferenças entre os estudos são designadas de heterogeneidade e a diversidade na característica da amostra é um aspecto bastante importante na interpretação dos resultados e pode estar associada à assimetria obtida no gráfico do "funil invertido", caracterizando viés nos estudos selecionados. Esse gráfico tem como premissa que o tamanho da amostra é o mais forte correlato do viés de publicação ${ }^{45}$. Outra questão que chama a atenção, nesse contexto da possível influência da característica da amostra na magnitude do efeito do resultado obtido, diz respeito aos diferentes grupos etários estudados. Em estudo transversal, realizado com 954 crianças e jovens belgas, de ambos os sexos, D'Hondt et al. ${ }^{27}$ encontraram uma relação inversa entre os valores do IMC e os resultados no KTK, mas que parece mais pronunciada nas idades maiores. Uma possível explicação para essa constatação pode ser a de que o desenvolvimento motor das crianças aumenta na medida em que tornam-se mais maduras. No entanto, após atingir à puberdade, esse aumento tende a ser mais lento e a estabilizar-se ${ }^{46}$. Por outro lado, o ganho de peso, que também ocorre com o avançar do estado maturacional, tende a aumentar ${ }^{47}$, o que contribuiria para uma maior chance de haver associação inversamente proporcional entre o IMC e o desempenho no KTK na puberdade e na adolescência, em relação à infância.

Chivers et al. ${ }^{48}$, em estudo publicado com 666 crianças e adolescentes do Western Australian Pregnancy Cohort (Raine) Study, com idades de 10 e 14 anos, agrupados em peso normal, sobrepeso e obesidade, cujo teste de habilidade motora foi o McCarron Assessment of Neuromuscular Development (MAND), não encontraram diferenças significativas nos participantes de desempenho motor global entre grupos nos sujeitos de 10 anos de idade, mas, aos 14 anos, o grupo com peso normal obteve melhores resultados do que o grupo de obesos.

Vale ressaltar, também, que nenhum dos estudos selecionados para a presente revisão contemplou em seus procedimentos metodológicos a avaliação do estado maturacional dos indivíduos da amostra. Tendo em vista a faixa etária dos sujeitos estudados e levando-se em consideração que o estado maturacional exerce efeito sobre o tamanho corporal de crianças e adolescentes ${ }^{46}$, torna-se evidente 
que esta é uma questão que pode reforçar a heterogeneidade metodológica dos estudos reportados e deve ser levada em consideração em estudos futuros.

\section{CONSIDERAÇÕES FINAIS}

O resultado global com base no modelo de efeitos fixos mostrou que a magnitude do efeito da associação entre o IMC e o desempenho no KTK foi classificada como pequena para o sexo masculino e moderada para o sexo feminino. Os estudos apresentaram características amostrais bastante divergentes quanto ao tamanho da amostra e a faixa etária dos sujeitos, além de nenhum deles ter analisado o estado maturacional dos seus participantes. Estes fatores reunidos podem explicar parte da heterogeneidade encontrada entre os estudos. Diante do exposto, conclui-se que há uma tendência de relação positiva entre maiores valores de IMC e menores resultados de de-

\section{REFERÊNCIAS}

1. Janssen I, Leblanc AG. Systematic review of the health benefits of physical activity and fitness in school-aged children and youth. Int J Behav Nutr Phys Act. 2010;7:40.

2. Gracia-Marco L, Vicente-Rodríguez G, Casajús JA, Molnar D, Castillo MJ, Moreno LA. Effect of fitness and physical activity on bone mass in adolescents: the HELENA Study. Eur J Appl Physiol. 2011;111(11):2671-80

3. Strong WB, Malina RM, Blimkie CJ, Daniels SR, Dishman RK, Gutin B, et al. Evidence based physical activity for school-age youth. J Pediatr. 2005;146(6):732-7.

4. Physical Activity Guidelines Advisory Committee report, 2008. To the Secretary of Health and Human Services. Part A: executive summary. Nutr Rev. 2009;67(2):114-20.

5. Twisk JW. Physical activity guidelines for children and adolescents: a critical review. Sports Med. 2001;31(8):617-27.

6. Ruiz JR, Ortega FB, Castillo R, Martín-Matillas M, Kwak L, Vicente-Rodríguez G, et al. Physical activity, fitness, weight status, and cognitive performance in adolescents. J Pediatr. 2010;157(6):917-922.e1-5.

7. Sayers A, Mattocks C, Deere K, Ness A, Riddoch C, Tobias JH. Habitual levels of vigorous, but not moderate or light, physical activity is positively related to cortical bone mass in adolescents. J Clin Endocrinol Metab. 2011;96(5):E793-802.

8. Adegboye AR, Anderssen SA, Froberg K, Sardinha LB, Heitmann BL, Steene-Johannessen J, et al. Recommended aerobic fitness level for metabolic health in children and adolescents: a study of diagnostic accuracy. Br J Sports Med. 2011;45(9):722-8.

9. Vasconcelos IQ, Stabelini Neto A, Mascarenhas LP, Bozza R, Ulbrich AZ, Campos Wd, Bertin RL. Cardiovascular risk factors in adolescents with different levels of energy expenditure. Arq Bras Cardiol. 2008;91(4):207-12, 227-33.

10. Graham DJ, Sirard JR, Neumark-Sztainer D. Adolescents' attitudes toward sports, exercise, and fitness predict physical activity 5 and 10 years later. Prev Med. 2011;52(2):130-2.

11. Hallal PC, Victora CG, Azevedo MR, Wells JC. Adolescent physical activity and health: a systematic review. Sports Med. 2006;36(12):1019-30.

12. Schilling VF. Kiphard EJ. The body coordination test (BCT). JOPERD. 1976;47(4):37.

13. Burns YR, Danks M, O'Callaghan MJ, Gray PH, Cooper D, Poulsen L, et al. Motor coordination difficulties and physical fitness of extremely-low-birthweight children. Dev Med Child Neurol. 2009;51(2):136-42.

14. Martins D, Maia J, Seabra A, Garganta R, Lopes V, Katzmarzyk P, et al. Correlates of changes in BMI of children from the Azores islands. Int J Obes (Lond). 2010;34(10):1487-93.

15. Lopes VP, Stodden DF, Bianchi MM, Maia JA, Rodrigues LP. Correlation between BMI and motor coordination in children. J Sci Med Sport. 2012;15(1):38-43.

16. Cairney J, Hay J, Veldhuizen S, Faught B. Comparison of VO2 maximum obtained from $20 \mathrm{~m}$ shuttle run and cycle ergometer in children with and without developmental coordination disorder. Res Dev Disabil. 2010;31(6):1332-9.

17. Machado-Rodrigues AM, Figueiredo AJ, Mota J, Cumming SP, Eisenmann JC, Malina RM, et al. Concurrent validation of estimated activity energy expenditure using a 3-day diary and accelerometry in adolescents. Scand J Med Sci Sports. 2012;22(2):259-64

18. Rivilis I, Hay J, Cairney J, Klentrou P, Liu J, Faught BE. Physical activity and fitness in children with developmental coordination disorder: a systematic review. Res Dev Disabil. 2011;32(3):894-910.

19. Krombholz H. Motor and cognitive performance of overweight preschool children. Percept Mot Skills. 2013;116(1):40-57.

20. Katzmarzyk PT, Malina RM, Beunen GP. The contribution of biological maturation to the strength and motor fitness of children. Ann Hum Biol. 1997;24(6):493-505.

21. Mutunga M, Gallagher AM, Boreham C, Watkins DC, Murray $\sqcup$, Cran G, et al. Socioeconomic differences in risk factors for obesity in adolescents in Northern Ireland. Int J Pediatr Obes. 2006;1 (2):114-9.

22. Okely AD, Booth ML, Patterson JW. Relationship of physical activity tofundamental movement skills among adolescents. Med Sci Sports Exerc. 2001;33(11):1899-904.

23. Lopes L, Santos R, Pereira B, Lopes VP. Associations between gross motor coordination and academic achievement in elementary school children. Hum Mov Sci. 2013;32(1):9-20.

24. Cools W, Martelaer KD, Samaey C, Andries C. Movement skill assessment of typically developing preschool children: a review of seven movement skill assessment tools. J Sports Sci Med. 2009:8:154-168 sempenho no KTK em crianças e adolescentes. No entanto, para confirmação desta tendência, recomenda-se para os próximos trabalhos que possibilitem uma interpretação isolada da relação existente entre o IMC e o KTK para ambos os sexos, em diferentes faixas etárias, além de incluírem em seus procedimentos metodológicos a avaliação do estado maturacional dos sujeitos da amostra.

\section{AGRADECIMENTOS}

Apoio da Coordenação de Aperfeiçoamento de Pessoal de Nível Superior (CAPES) na forma de bolsa de estudos, processo número: 1617/13-3.

Todos os autores declararam não haver qualquer potencial conflito de interesses referente a este artigo.
25. Kiphard EJ, Schilling F. Kör perkoordinationstest für Kinder - Body coordination test for children Weinheim, Germany: Beltz Test GmbH; 1974.

26. Smits-engelsman BCM, Henderson SE. The assessment of children with developmental coordination disorders in the Netherlands : The relationship between the movement assessment battery for children and the Korperkoordinations test fur Kinder. Human Movement Science. 1998;17:699-709.

27. D'Hondt E, Deforche B, Vaeyens R, Vandorpe B, Vandendriessche J, Pion J, et al. Gross motor coordination in relation to weight status and age in 5- to 12-year-old boys and girls: a cross-sectional study. Int J Pediatr Obes. 2011:6(2-2):e556-64.

28. D'Hondt E, Deforche B, Gentier I, Verstuyf J, Vaeyens R, De Bourdeaudhuij I, et al. A longitudinal study of gross motor coordination and weight status in children. Obesity (Silver Spring). 2014;22(6):1505-11.

29. Lubans DR, Morgan PJ, Cliff DP, Barnett LM, Okely AD. Fundamental movement skills in children and adolescents: review of associated health benefits. Sports Med. 2010;40(12):1019-35.

30. Maher CG, Sherrington C, Herbert RD, Moseley AM, Elkins M. Reliability of the PEDro scale for rating quality of randomized controlled trials. Phys Ther. 2003;83(8):713-21.

31. Verhagen AP, de Vet HC, de Bie RA, Kessels AG, Boers M, Bouter LM, et al. The Delphi list: a criteria list for quality assessment of randomized clinical trials for conducting systematic reviews developed by Delphi consensus. J Clin Epidemiol. 1998;51(12):1235-41.

32. Moseley AM, Herbert RD, Sherrington C, Maher CG. Evidence for physiotherapy practice: a survey of the Physiotherapy Evidence Database (PEDro). Aust J Physiother. 2002;48(1):43-9.

33. von Elm E, Altman DG, Egger M, Pocock SJ, Gøtzsche PC, Vandenbroucke JP. The Strengthening the Reporting of Observational Studies in Epidemiology (STROBE) statement: guidelines for reporting observational studies. J Clin Epidemiol. 2008 Apr:61(4):344-9.

34. Malta M, Cardoso LO, Bastos Fl, Magnanini MM, Silva CM. STROBE initiative: guidelines on reporting observational studies. Rev Saude Publica. 2010;44(3):559-65.

35. Harris RJ, Bradburn MJ, Deeks JJ, Altman DG, Harbord RM, Sterne JAC. Metan: Fixed- and random-effects meta-analysis. Stata J. 2008;8:3-28.

36. Hopkins WG, Marshall SW, Batterham AM, Hanin J. Progressive statistics for studies in sports medicine and exercise science. Med Sci Sports Exerc. 2009;41(1):3-13.

37. Sterne J. Meta-Anaysis in Stata: an updated collection from Stata Journal. Texas, USA: Statapress; 2009

38. Catenassi FZ, Marques I, Bastos CB, Basso L, Ronque ERV, Gerage AM. Relação entre índice de mas sa corporal e habilidade motora grossa em crianças de quatro a seis anos. Rev Bras Med Esporte. 2007:13(4):227-30

39. D'Hondt E, Gentier I, Deforche B, Tanghe A, De Bourdeaudhuij I, Lenoir M. Weight loss and improved gross motor coordination in children as a result of multidisciplinary residential obesity treatment. Obesity (Silver Spring). 2011;19(10):1999-2005.

40. Graf C, Koch B, Kretschmann-Kandel E, Falkowski G, Christ H, Coburger S, et al. Correlation between BMI, leisure habits and motor abilities in childhood (CHILT-project). Int J Obes Relat Metab Disord. 2004;28(1):22-6.

41. Egger M, Smith GD. Bias in location and selection of studies. BMJ. 1998;316(7124):61-6.

42. Melo MM, Lopes VP. Associação entre o índice de massa corporal e a coordenação motora em crianças. Rev Bras Educ Fis Esporte. 2013;27(1):7-13.

43. Lopes L, Santos R, Moreira C, Pereira B, Lopes VP. Sensitivity and specificity of different measures of adiposity to distinguish between low/high motor coordination. J Pediatr. 2015;91(1):44-51.

44. D'Hondt E, Deforche B, Gentier I, De Bourdeaudhuij I, Vaeyens R, Philippaerts R, et al. A longitudina analysis of gross motor coordination in overweight and obese children versus normal-weight peers. Int J Obes (Lond). 2013:37(1):61-7.

45. Moayyedi P. Meta-analysis: Can we mix apples and oranges? Am J Gastroenterol. 2004;99(12):2297-301. 46. Malina RM, Bouchard C, Bar-Or O. Growth, maturation, and physical activity. Champaign, IL: Human Kinetics; 2004.

47. World Health Organisation (WHO). Prevalence of excess body weight and obesity in children and adolescents. Geneva: WHO; 2007.

48. Chivers P, Larkin D, Rose E, Beilin L, Hands B. Low motor performance scores among overweight children: poor coordination or morphological constraints? Hum Mov Sci. 2013;32(5):1127-37. 